\title{
Hippocampal Function in Healthy Carriers of the $C L U$ Alzheimer's Disease Risk Variant
}

\author{
Susanne Erk, ${ }^{1 \star}$ Andreas Meyer-Lindenberg, ${ }^{7 \star}$ Carola Opitz von Boberfeld, ${ }^{2}$ Christine Esslinger, ${ }^{10}$ Knut Schnell, ${ }^{11}$ Peter \\ Kirsch, ${ }^{9}$ Manuel Mattheisen, ${ }^{4,6,13}$ Thomas W. Mühleisen, ${ }^{4,5}$ Sven Cichon, ${ }^{4,5,12}$ Stephanie H. Witt, ${ }^{8}$ Marcella Rietschel, ${ }^{8}$ \\ Markus M. Nöthen, ${ }^{4,5}$ and Henrik Walter ${ }^{1,2,3}$ \\ ${ }^{1}$ Division of Mind and Brain Research, Department of Psychiatry and Psychotherapy, Charité-Universitätsmedizin Berlin, Campus Mitte, D-11017 Berlin, \\ Germany, ${ }^{2}$ Division of Medical Psychology, ${ }^{3}$ Department of Psychiatry and Psychotherapy, ${ }^{4}$ Department of Genomics, Life and Brain Center, ${ }^{5}$ Institute of \\ Human Genetics, and ${ }^{~ I n s t i t u t e ~ o f ~ M e d i c a l ~ B i o m e t r y, ~ I n f o r m a t i c s, ~ a n d ~ E p i d e m i o l o g y, ~ U n i v e r s i t y ~ o f ~ B o n n, ~ D-53115 ~ B o n n, ~ G e r m a n y, ~}{ }^{7}$ Department of \\ Psychiatry and Psychotherapy, ${ }^{8}$ Department of Genetic Epidemiology in Psychiatry, and ${ }^{9}$ Department of Psychology, Central Institute of Mental Health, \\ D-68159 Mannheim, Germany, ${ }^{10}$ Department of Neurology, Otto-von-Guericke Universität, D-39120 Magdeburg, Germany, ${ }^{11}$ Department of General \\ Psychiatry, University of Heidelberg, D-69117 Heidelberg, Germany, ${ }^{12}$ Institute of Neuroscience and Medicine, Research Centre Jülich, D-52425 Jülich, \\ Germany, and ${ }^{13}$ Department of Biostatistics, Harvard School of Public Health, Boston, Massachusetts 02115
}

Alzheimer's disease is a devastating, common, progressive dementia with considerable heritability. Recently, a genetic variant associated with the disease was discovered at $C L U$ (rs11136000) with genome-wide support. Here we show, using an imaging genetics approach in a large genotyped sample, that healthy carriers of the variant exhibit altered coupling between hippocampus and prefrontal cortex during memory processing, mirroring clinical evidence of disturbed connectivity in patients and providing a neurogenetic mechanism for CLU-associated risk and protection.

\section{Introduction}

Alzheimer's disease (AD) is the most common form of dementia and one of the most feared concomitants of aging. AD has significant heritability (between 60 and 80\%) (Gatz et al., 2006) and affects nearly $10 \%$ of individuals at the age of 65 years and almost $50 \%$ of individuals who reach their nineties (Evans et al., 1989). Characterized first by memory loss, the disease progresses to cognitive decline and incapacity. To date, no cure for this disease is available; however, new treatments are underway and there is an increasing push to identify the earliest disease stages and those individuals at risk who will benefit most from disease-modifying interventions. Neuroimaging work in patients has demonstrated reduced activation of the hippocampal formation during memory encoding and retrieval as well as altered functional coupling between hippocampus and the dorsolateral prefrontal cortex (DLPFC) (Grady et al., 2001; Allen et al., 2007; Dickerson and Sperling, 2008), but the relevance of these findings for heritable risk was undetermined. Previous genetic studies have led to the

Received Sept. 29, 2011; accepted 0ct. 26, 2011.

Author contributions:S.E., A.M.-L., K.S., and H.W. designed research;S.E., C.O.v.B., C.E., K.S., and P.K. performed research; C.E., P.K., and M.M. contributed unpublished reagents/analytic tools; S.E., C.O.v.B., M.M., T.W.M., S.C., S.H.W., M.R., and M.M.N. analyzed data; S.E., A.M.-L., and H.W. wrote the paper.

This work was supported by Bundesministerium fur Bildung und Forschung (NGFNplus MooDs) and Deutsche Forschungsgemeinschaft (SFB 636-B7). We thank Claudia Arnold, Leila Haddad, Beate Newport, Dagmar Gass, Daniela Mier, Carina Sauer, and Kyeon Raab for help with data acquisition.

*S.E. and A.M.-L. contributed equally to this work.

Correspondence should be addressed to Dr. Susanne Erk, Division of Mind and Brain Research, Department of Psychiatry and Psychotherapy, Charité-Universitätsmedizin Berlin, Charitéplatz 1, D-11017 Berlin, Germany. Email: susanne.erk@charite.de.

DOI:10.1523/JNEUROSCI.4960-11.2011

Copyright $\odot 2011$ the authors $\quad 0270-6474 / 11 / 3118180-05 \$ 15.00 / 0$ identification of APOE4 as an unequivocal susceptibility locus for late-onset AD (Farrer et al., 1997), with neuroimaging studies in APOE4 carriers suggesting compensatory increased recruitment of hippocampal and prefrontal regions (Bookheimer and Burggren, 2009) and evidence for disturbed white matter integrity (Honea et al., 2009; Sheline et al., 2010). Other genes, however, were less consistently implied. In 2009, through genome-wide association study, a single nucleotide polymorphism (SNP) within clusterine ( $C L U$, also called $A P O J)$ on chromosome $8 \mathrm{p} 21.1$, rs 11136000 was discovered to be significantly associated with AD in two independent studies (Harold et al., 2009; Lambert et al., 2009). This finding has since been replicated by several groups (Carrasquillo et al., 2010; Corneveaux et al., 2010; Jun et al., 2010; Kamboh et al., 2010; Seshadri et al., 2010), leading to $C L U$ being the third top-ranking locus associated with Alzheimer's disease in the AlzGene database (http://www.alzgene.org). Carrying the minor allele $\mathrm{T}$ is assumed to establish a protective effect and reduces the risk to develop AD by $16 \%$ (Bertram and Tanzi, 2010); conversely, carrying the C-allele could be interpreted as an $\mathrm{AD}$ risk factor. As the earliest signs of $\mathrm{AD}$ pathology appear in the hippocampus and entorhinal cortex, these data provide a clear a priori hypothesis that genetic risk for Alzheimer's disease in CLU could be neurally mediated through hippocampal dysfunction, especially during episodic memory, which is affected early in the course of AD.

Recently, evidence for the impact of CLU rs11136000 on brain phenotypes has been provided using diffusion tensor imaging showing lower white matter integrity in carriers of the rs11136000-C risk allele (Braskie et al., 2011) and using functional magnetic resonance imaging (fMRI) showing compen- 
Table 1. Sample characteristics: distribution of different parameters for the three allele types of rs 11136000

\begin{tabular}{lllcc}
\hline & CC & CT & TT & P \\
\hline Subjects & 36 & 52 & 21 & \\
Site (Bonn/Mannheim) & $17 / 19$ & $23 / 29$ & $10 / 11$ & $6 / 15$ \\
Sex (male/female) & $19 / 17$ & $23 / 29$ & $19 / 2$ & 0.947 \\
Handedness (right/left) & $33 / 3$ & $49 / 3$ & $33.29 \pm 11.2$ & 0.206 \\
Age (years) & $30.50 \pm 10.3$ & $33.98 \pm 9.6$ & $6.57 \pm 0.8$ & 0.393 \\
School education (years) & $6.36 \pm 1.1$ & $5.94 \pm 1.3$ & $3 / 18$ & 0.277 \\
APOE status (APOE4 & & $17 / 35$ & 0.073 \\
\hline
\end{tabular}

$C$, Cytosine; $T$, thymine, protective allele; $p, p$ value $\pm S D$.

satory hyperactivation of frontal and medial temporal lobe regions in risk allele carriers during a working memory task (Lancaster et al., 2011).

To test for the functional relevance of CLU rs11136000 during episodic memory, a process mediated through hippocampal function and crucially affected by $\mathrm{AD}$, we used fMRI in healthy participants genotyped for rs11136000 and a task optimized for imaging genetics that allows probing of different cognitive subprocesses of episodic memory, i.e., encoding, recall, and recognition of associative episodic information (Erk et al., 2010, 2011). We studied task-related regional activation as well as functional coupling, using functional connectivity, a well established method that measures correlation between BOLD time series in different brain regions that has been used successfully to delineate the functional anatomy of brain interactions and the impact of genetic variation (Esslinger et al., 2009).

\section{Materials and Methods}

Subjects. Healthy German volunteers with grandparents of European ancestry were recruited at Bonn (50 volunteers) and Mannheim (59 volunteers) as part of an ongoing study on neurogenetic mechanisms of psychiatric disease (Esslinger et al., 2009). All subjects gave written informed consent to the study. No participant had lifetime or family history of psychiatric diseases. Thirty-six subjects were rs111136000 CC homozygotes, 52 were CT heterozygotes, and 21 were TT homozygotes. The allele frequencies were in Hardy-Weinberg equilibrium $\left(\chi^{2}=0.27\right.$, $\mathrm{df}=1, p=0.60)$. Genotype distributions did not differ between sites ( $p=0.947)$. Gender, age, handedness, and level of education did not differ significantly between genotype groups (Table 1). We additionally identified APOE status of the participants. APOE genotype distribution did not differ between the three rs 111136000 allele groups (Table 1). The study was approved by the local ethics committees of the universities of Bonn and Heidelberg.

DNA extraction and genotyping. EDTA anti-coagulated venous blood samples were collected from all individuals. Lymphocyte DNA was isolated using the Chemagic Magnetic Separation Module I (Chemagen) according to the manufacturer's recommendations. Genotype data for rs11136000 were extracted from a genome-wide dataset generated at the Department of Genomics, Life and Brain Center, University of Bonn using Human610-Quad Bead Arrays (Illumina). For identification of APOE status, rs429358 and rs7412 were genotyped on the MALDI TOFbased MassARRAY system using the iPLEX Gold assay (Sequenom). The iPLEX primer sequences and assay conditions may be obtained from the authors upon request.

Functional imaging task. During fMRI scanning, subjects completed three consecutive blocks of memory tasks, based on a paradigm previously used for imaging genetics, as part of a functional imaging genetics battery (Erk et al., 2010, 2011). The encoding task consisted of 16 face-profession pairs and 24 head contours with four blocks of four face-profession pairs and four blocks of six head contours each. Faceprofession pairs were presented for $6 \mathrm{~s}$, and head contour for $4 \mathrm{~s}$. Thus, each block lasted $24 \mathrm{~s}$. Participants had to imagine the person acting in a scene of the written profession and had to indicate whether the profession suited the presented face. During the control condition, participants had to indicate which ear of the depicted head contour was larger. Each block was presented twice. During recall, faces were presented together with the question whether the depicted person had to complete apprenticeship or academic studies to qualify for the respective profession that had been learned during encoding. Stimulus duration and control condition were similar to encoding. Blocks were presented only once. Note that completion of this task also required making a decision about the educational training the profession requires, and thus also invoked other cognitive processes. However, the main task was to freely recall the profession learned during the encoding task. Only then could an answer be given. For recognition testing, faces were depicted together with two written professions and subjects had to indicate which profession was correct. Stimulus duration for recognition was $3 \mathrm{~s}$. The control condition consisted of four blocks of four head contours each $(3 \mathrm{~s})$. Thus, each recognition block lasted $12 \mathrm{~s}$. As in the recall tasks, blocks were only presented once.

Neuropsychological measures. On the second day, subjects underwent neuropsychological assessment, including tests for verbal intelligence [Mehrfachwahl-Wortschatz-Intelligenztest (MWT-B)], memory [Verbaler Lern und Merkfaehigkeits-Test (VLMT), translated verbal version of Rey auditory learning task], selective attention (D2), and working memory (Digit Span) reported here.

Imaging parameters. BOLD fMRI was performed on two Siemens Trio 3T scanners at the Central Institute of Mental Health Mannheim and the University of Bonn. At both sites, identical sequences and scanner protocols were used [parameters: 33 slices; axially tilted $\left(-30^{\circ}\right)$; slice thickness, $2.4 \mathrm{~mm}+0.6 \mathrm{~mm}$ gap; FOV, $192 \mathrm{~mm}$; TR, $1.96 \mathrm{~s}$; TE, 30; flip angle, $80^{\circ}$. Quality assurance (QA) measures were conducted on every measurement day at both sites according to a multicenter QA protocol revealing stable signals over time (Friedman and Glover, 2006; Erk et al., 2010). To stringently account for any differences in signal-to-noise across sites, site was used as a confounding covariate for all statistical analyses. Furthermore, in post hoc testing, we examined by ANOVA whether scanner site by genotype interactions were found in our anatomical regions of interest (ROI). No significant interactions were found.

Functional image processing. Image processing and statistical analyses were conducted using statistical parametric mapping methods as implemented in SPM5 (http://www.fil.ion.ucl.ac.uk/spm/software/spm5/) and were similar for all tasks. Briefly, images were realigned to a mean image (movement parameters were confined to $<3 \mathrm{~mm}$ translation and $<3^{\circ}$ rotation between volumes), slice time corrected, spatially normalized to a standard stereotactic space (a brain template created by the Montreal Neurological Institute) with volume units (voxels) of $2 \times 2 \times$ $2 \mathrm{~mm}$, smoothed with an $8 \mathrm{~mm}$ FWHM Gaussian filter, and ratio normalized to the whole-brain global mean. A first-level fixed-effects model was computed for each participant. Regressors were created from the time course of the two experimental conditions (memory, control) and convolved with a canonical hemodynamic response function. Movement parameters were included in the first-level model as regressors of no interest. For each subject, statistical contrast images of memory versus control were obtained. To test for genetic association, these were analyzed using the general linear model in a second-level multiple regression random-effects analysis with scanner site as nuisance covariate to identify genotype effects on activation or connectivity. Because CLU has biochemically similar functionalities to APOE, both variants are associated with $\mathrm{AD}$, and previous neuroimaging studies have shown altered brain function in carriers of the APOE4 allele, we examined for a possible 
additive or interactive effect of these genotypes, including APOE status and interaction with CLU as covariates.

Functional connectivity analyses. Analyses to measure functional connectivity used a seed region approach, as described previously (Esslinger et al., 2009). For each subject, time series were extracted from the left and right hippocampus using first eigenvariates from all voxels within the respective hippocampus mask. Following our previous practice (Esslinger et al., 2009), to derive a robust summary measure of activity in the respective hippocampus ROI, we excluded white matter by restricting the averaging to voxels related to task at a $p<00.5$ level (note that this level was not used for statistical inference). Using SPM5, seed time series were high-pass filtered (128 s) and task-related variance was removed to avoid measuring coactivation that was solely due to temporal correlation with the experimental paradigm. To account for unspecific noise, first eigenvariates from masks covering CSF and white matter were extracted for each individual and entered, together with movement covariates and task regressors, into whole-brain multiple regression analyses where the respective seed region time series, i.e., the time series from the right or left hippocampus, was the covariate of interest. Thus, we identified voxels whose activity show significant covariation with the left or right hippocampus. Here, two brain areas are called functionally connected if their BOLD signal time series covary over time. The resulting maps of partial correlation with the left or right hippocampal seed region were then each subjected to a random-effects analysis in SPM5 using multiple regression randomeffects analysis as described above with scanning site as nuisance covariate; in a second analysis, $A P O E$ status and interaction with $C L U$ variants were used as additional covariates.

Statistical inference. For all imaging methods, the significance threshold was set to $p<0.05$, corrected for multiple comparisons across the whole brain using family-wise error rate, which strongly controls for type I error in imaging genetics. The hippocampus ROI for seed voxel extraction was defined a priori and created using anatomical labels provided by the Wake Forest University PickAtlas (www.fmri.wfubmc.edu/ downloads).

\section{Results}

Regional brain activation during each of the three episodic memory tasks was not related to genotype, but coupling between hippocampus and DLPFC was significantly altered (Fig. 1). During recall, subjects carrying the C-risk allele exhibited allele dose-dependent reduced coupling between right hippocampus and right DLPFC $(x=32, y=52, z=20, Z=5.06)$. Likewise, during recognition, the risk allele conferred allele dose-dependent decreased connectivity between left hippocampus and right DLPFC $(x=36, y=34, z=24$, $Z=4.73$ ). Results did not change significantly when $A P O E$ status was included as covariate (recall: $x=32, y=52, z=20, Z=5.05$; recognition: $x=36, y=34, z=24, Z=4.68$ ) and there was no significant interaction between CLU and APOE status. rs11136000 genotypes had no impact on task performance or neuropsychological memory testing (Table 2). This can be expected from the concept of intermediate phenotypes (Meyer-Lindenberg and
Coupling with right Hippocampus

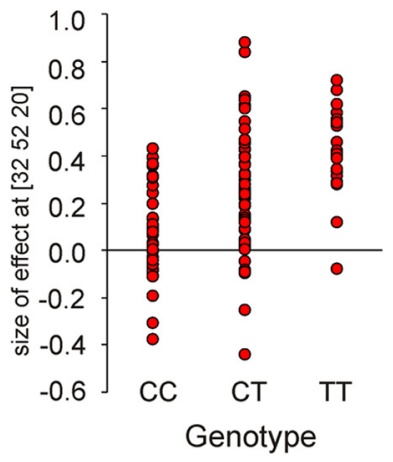

Coupling with left Hippocampus

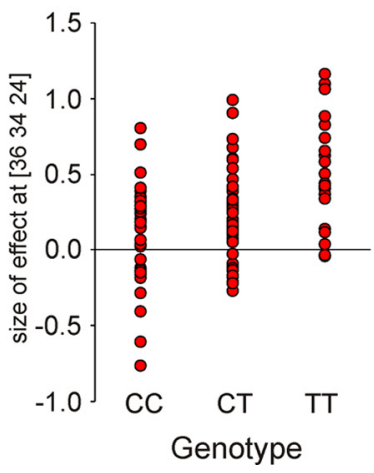

Figure 1. Altered functional coupling of the hippocampus in carriers of the risk variant for CLU rs11136000. A, Carriers of the C-risk allele exhibit significantly decreased allele dosage-dependent coupling of the right DLPFC with the right hippocampus seed (t) 列 red dot represents size of effect in one subject and reflects connectivity between right DLPFC and left hippocampal seed region. $C$, Cytosine; T, thymine. Number of subjects in each genotype group: $\mathrm{CC}, n=36 ; \mathrm{CT}, n=52 ; \mathrm{TT}, n=21$.

Weinberger, 2006), indicating that genetic variation is more penetrant on the neurobiological phenotype level than in behavior and showing that, while carrier status for rs11136000 already induces neurofunctional alterations in healthy carriers, it is not enough to behaviorally impair memory function. To test for a link between genotype-dependent differences in connectivity and behavioral measures, we extracted individual beta weights from the peakactivated voxel in the right DLPFC. The beta weights were externally correlated (SPSS statistical software, version 17; SPSS) with individual scores in tests for verbal intelligence (MWT-B), episodic memory (VLMT), selective attention (D2), and working memory (Digit Span), as well as recall and recognition performance during scanning. No significant correlations between behavioral measures and functional coupling were observed.

\section{Discussion}

Recent genome-wide association studies have identified a SNP (11136000) within the Clusterin gene as strongly associated with risk for Alzheimers disease (Harold et al., 2009; Lambert et al., 2009; Carrasquillo et al., 2010; Corneveaux et al., 2010; Jun et al., 2010; Kamboh et al., 2010; Seshadri et al., 2010). $C L U$ appears to be a biologically plausible candidate for $\mathrm{AD}$ as it binds amyloid beta peptide $(\mathrm{A} \beta)$ and critically modifies $\mathrm{A} \beta$ 
Table 2. Behavioral data

\begin{tabular}{|c|c|c|c|c|}
\hline & $\mathrm{CC}$ & CT & TT & $p$ \\
\hline Subjects & 36 & 52 & 21 & \\
\hline Recall (hits) & $11.19 \pm 1.7$ & $11.31 \pm 1.8$ & $12.05 \pm 1.5$ & 0.162 \\
\hline Recognition (hits) & $12.61 \pm 2.3$ & $12.48 \pm 2.5$ & $13.19 \pm 2.0$ & 0.498 \\
\hline VLMT (hits late recall) & $12.6 \pm 2.7$ & $12.96 \pm 2.6$ & $13.24 \pm 1.8$ & 0.650 \\
\hline VLMT (hits recognition) & $14.60 \pm 0.8$ & $14.29 \pm 1.2$ & $14.71 \pm 0.6$ & 0.197 \\
\hline MWT-B (score) & $30.40 \pm 3.0$ & $29.38 \pm 5.1$ & $31.10 \pm 2.5$ & 0.244 \\
\hline Digit Span forwards & $8.73 \pm 2.3$ & $8.38 \pm 2.2$ & $9.00 \pm 1.9$ & 0.519 \\
\hline Digit Span backwards & $7.87 \pm 2.6$ & $7.65 \pm 2.1$ & $7.76 \pm 2.0$ & 0.912 \\
\hline
\end{tabular}

Task performance, scores in a verbal episodic memory task, verbal intelligence, selective attention, and working memory did not differ significantly between genotype groups. C, Cytosine; T, thymine, protective allele; $p, p$ value \pm SD; VLMT, Verbal Learning and Memory Test (translated verbal version of Rey Auditory Learning Task); MWT-B, Test of verbal intelligence; D2, Test of selective attention (TN-E, total performance - errors; (P, index of concentration performance).

clearance from the brain across the blood-brain barrier (Bell et al., 2007).

To date, no functional effects of $C L U$ variants on $\mathrm{AD}$ are known (Sleegers et al., 2010) and it is of utmost relevance to identify the underlying mechanisms of the association between genetic markers and the disease phenotype. This challenge leads to the strategy of studying underlying quantitative traits that are more directly related to biology, namely brain activation as intermediate phenotype in an imaging genetics approach. This is with the assumption that genes will show higher penetrance for brain-activation phenotypes closer to gene biology than behavioral or clinical measures (MeyerLindenberg and Weinberger, 2006).

Here, we investigated the neurogenetic mechanisms for $\mathrm{AD}$ associated with rs111136000, using an imaging genetics approach with brain activation during episodic memory as an intermediate phenotype. We observed significantly reduced coupling between hippocampus and DLPFC during episodic memory retrieval, i.e., recall as well as recognition of associative information. Because rs11136000 has been strongly associated with AD at the genomewide level, our findings identify altered connectivity as a neural mechanism that can be linked to a disease architecture, where carrying the risk variant predicted reduced coupling between hippocampus and DLPFC that could contribute to risk of developing AD. Our findings appear to be independent of the individual $A P O E$ status, as our results remain significant when including $A P O E$ as a covariate and no interaction between $C L U$ variant and $A P O E$ status was observed. Interestingly, although $C L U$ and $A P O E$ have similar functionality, no significant interaction of $C L U$ with presence or absence of APOE4 alleles has been observed in population genetics (Harold et al., 2009; Jun et al., 2010). Only in one study was a more strongly significant association between $C L U$ and $\mathrm{AD}$ in $A P O E 4$ carriers reported, although the association was significant in both groups (Lambert et al., 2009).

The functional relevance of our finding of altered connectivity is indicated by cognitive neuroscience evidence showing that the prefrontal cortex and the medial temporal lobe (MTL) form part of a distributed functional network of regions involved in memory, and that interactions between these regions are particularly important for retrieval operations (Frankland and Bontempi, 2005). Coupling between the prefrontal cortex and the MTL may facilitate focused attention on behaviorally relevant stimuli processed through reciprocal pathways between these regions (Rempel-Clower and Barbas, 2000). It has been proposed that prefrontal-hippocampal interactions are particularly crucial during episodic memory retrieval and are modulated by cognitive effort during retrieval attempt (Schacter et al., 1996; Simons and Spiers,
2003), with the DLPFC being involved in verification and monitoring of recollected information (Burgess and Shallice, 1996).

Reduced coupling between hippocampus and DLPFC as a risk neural mechanism is further backed by clinical evidence, reporting reduced or even absent hippocampal-prefrontal connectivity during memory retrieval in AD patients (Grady et al., 2001; Allen et al., 2007), as well as subjects suffering from amnestic mild cognitive impairment (MCI) (Bai et al., 2009), an intermediate stage between normal aging and dementia. It has been suggested that memory breakdown in $\mathrm{AD}$ is related to a marked reduction of integrated activity within a distributed network, including hippocampus and DLPFC (Grady et al., 2001). Further support for the relevance of hippocampal-prefrontal interaction in AD risk was given by a recent study by our group, where we demonstrated that subjective memory impairment - an early, pre-MCI stage in the continuum of Alzheimer's disease manifestation-is characterized by early hippocampal dysfunction and increased compensatory effort, reflected in DLPFC activation, to enable sustained performance in the presence of early pathology (Erk et al., 2011). A recent fMRI study provided evidence for aberrant activation in the same regions, namely DLFPC and hippocampus, during working memory performance in a small sample of healthy young individuals genotyped for rs11136000 (Lancaster et al., 2011). These results, although in a different cognitive domain, additionally emphasize the relevance of prefrontal-hippocampal interaction in $\mathrm{AD}$ risk pathology. The recently reported finding of disturbed white matter integrity in healthy carriers of the rs11136000 risk variant (Braskie et al., 2011) points to neurodevelopmental vulnerability and further supports the idea that brain connectivity could mediate genetic risk for a behavioral phenotype, possibly to a higher degree than regional brain activation (Meyer-Lindenberg, 2009).

The molecular and cellular mechanisms leading to the observed changes in connectivity remain to be elucidated. However, our data show that CLU rs11136000 itself (or a hitherto unknown variant in linkage disequilibrium with it) is functional in the human brain even in the absence of overt disease or cognitive impairment, and provide a mechanism for genetic findings supported by genome-wide association that are congruent with current findings on the pathophysiology of overt disease. Neurobiologically, our results show that altered connectivity contributes to the neurogenetic architecture implicated in Alzheimer's disease susceptibility. To the degree that new potentially curative therapies are being developed and tested, early detection of disease susceptibility is of crucial importance, particularly as life span increases and thus the incidence for late-onset AD. Since our current findings track 
an established genetic risk factor in the absence of clinical symptoms, they might be useful as a biomarker to stratify and monitor treatment studies and might even identify a novel potential therapeutic target for this devastating disease.

\section{References}

Allen G, Barnard H, McColl R, Hester AL, Fields JA, Weiner MF, Ringe WK, Lipton AM, Brooker M, McDonald E, Rubin CD, Cullum CM (2007) Reduced hippocampal functional connectivity in Alzheimer disease. Arch Neurol 64:1482-1487.

Bai F, Zhang Z, Watson DR, Yu H, Shi Y, Yuan Y, Zang Y, Zhu C, Qian Y (2009) Abnormal functional connectivity of hippocampus during episodic memory retrieval processing network in amnestic mild cognitive impairment. Biol Psychiatry 65:951-958.

Bell RD, Sagare AP, Friedman AE, Bedi GS, Holtzman DM, Deane R, Zlokovic BV (2007) Transport pathways for clearance of human Alzheimer's amyloid beta-peptide and apolipoproteins $\mathrm{E}$ and $\mathrm{J}$ in the mouse central nervous system. J Cereb Blood Flow Metab 27:909-918.

Bertram L, Tanzi RE (2010) Alzheimer disease: new light on an old CLU. Nat Rev Neurol 6:11-13.

Bookheimer S, Burggren A (2009) APOE-4 genotype and neurophysiological vulnerability to Alzheimer's and cognitive aging. Annu Rev Clin Psychol 5:343-362.

Braskie MN, Jahanshad N, Stein JL, Barysheva M, McMahon KL, de Zubicaray GI, Martin NG, Wright MJ, Ringman JM, Toga AW, Thompson PM (2011) Common Alzheimer's disease risk variant within the CLU gene affects white matter microstructure in young adults. J Neurosci 31:6764-6770.

Burgess PW, Shallice T (1996) Confabulation and the control of recollection. Memory 4:359-411.

Carrasquillo MM, Belbin O, Hunter TA, Ma L, Bisceglio GD, Zou F, Crook JE, Pankratz VS, Dickson DW, Graff-Radford NR, Petersen RC, Morgan K, Younkin SG (2010) Replication of CLU, CR1, and PICALM associations with Alzheimer disease. Arch Neurol 67:961-964.

Corneveaux JJ, Myers AJ, Allen AN, Pruzin JJ, Ramirez M, Engel A, Nalls MA, Chen K, Lee W, Chewning K, Villa SE, Meechoovet HB, Gerber JD, Frost D, Benson HL, O’Reilly S, Chibnik LB, Shulman JM, Singleton AB, Craig DW, et al. (2010) Association of CR1, CLU and PICALM with Alzheimer's disease in a cohort of clinically characterized and neuropathologically verified individuals. Hum Mol Genet 19:3295-3301.

Dickerson BC, Sperling RA (2008) Functional abnormalities of the medial temporal lobe memory system in mild cognitive impairment and Alzheimer's disease: insights from functional MRI studies. Neuropsychologia 46:1624-1635.

Erk S, Meyer-Lindenberg A, Schnell K, Opitz von Boberfeld C, Esslinger C, Kirsch P, Grimm O, Arnold C, Haddad L, Witt SH, Cichon S, Nöthen MM, Rietschel M, Walter H (2010) Brain function in carriers of a genome-wide supported bipolar disorder variant. Arch Gen Psychiatry 67:803-811.

Erk S, Spottke A, Meisen A, Wagner M, Walter H, Jessen F (2011) Evidence for neuronal compensation during episodic memory in subjective memory impairment. Arch Gen Psychiatry 68:845-852.

Esslinger C, Walter H, Kirsch P, Erk S, Schnell K, Arnold C, Haddad L, Mier D, Opitz von Boberfeld C, Raab K, Witt SH, Rietschel M, Cichon S, Meyer-Lindenberg A (2009) Neural mechanisms of a genome-wide supported psychosis variant. Science 324:605.

Evans DA, Funkenstein HH, Albert MS, Scherr PA, Cook NR, Chown MJ, Hebert LE, Hennekens CH, Taylor JO (1989) Prevalence of Alzheimer's disease in a community population of older persons: higher than previously reported. JAMA 262:2551-2556.

Farrer LA, Cupples LA, Haines JL, Hyman B, Kukull WA, Mayeux R, Myers RH, Pericak-Vance MA, Risch N, van Duijn CM (1997) Effects of age, sex, and ethnicity on the association between apolipoprotein E genotype and Alzheimer disease: a meta-analysis. APOE and Alzheimer Disease Meta Analysis Consortium. JAMA 278:1349-1356.
Frankland PW, Bontempi B (2005) The organization of recent and remote memories. Nat Rev Neurosci 6:119-130.

Friedman L, Glover GH (2006) Report on a multicenter fMRI quality assurance protocol. J Magn Reson Imaging 23:827-839.

Gatz M, Reynolds CA, Fratiglioni L, Johansson B, Mortimer JA, Berg S, Fiske A, Pedersen NL (2006) Role of genes and environments for explaining Alzheimer disease. Arch Gen Psychiatry 63:168-174.

Grady CL, Furey ML, Pietrini P, Horwitz B, Rapoport SI (2001) Altered brain functional connectivity and impaired short-term memory in Alzheimer's disease. Brain 124:739-756.

Harold D, Abraham R, Hollingworth P, Sims R, Gerrish A, Hamshere ML, Pahwa JS, Moskvina V, Dowzell K, Williams A, Jones N, Thomas C, Stretton A, Morgan AR, Lovestone S, Powell J, Proitsi P, Lupton MK, Brayne C, Rubinsztein DC, et al. (2009) Genome-wide association study identifies variants at CLU and PICALM associated with Alzheimer's disease. Nat Genet 41:1088-1093.

Honea RA, Vidoni E, Harsha A, Burns JM (2009) Impact of APOE on the healthy aging brain: a voxel-based MRI and DTI study. J Alzheimers Dis 18:553-564.

Jun G, Naj AC, Beecham GW, Wang LS, Buros J, Gallins PJ, Buxbaum JD, Ertekin-Taner N, Fallin MD, Friedland R, Inzelberg R, Kramer P, Rogaeva E, St George-Hyslop P, St George-Hyslop P, Cantwell LB, Dombroski BA, Saykin AJ, Reiman EM, Bennett DA, et al. (2010) Meta-analysis confirms CR1, CLU, and PICALM as alzheimer disease risk loci and reveals interactions with APOE genotypes. Arch Neurol 67:1473-1484.

Kamboh MI, Minster RL, Demirci FY, Ganguli M, Dekosky ST, Lopez OL, Barmada MM (2010) Association of CLU and PICALM variants with Alzheimer's disease. Neurobiol Aging. Advance online publication. Retrieved June 2010. doi:10.1016/j.neurobiolaging.2010.04.015.

Lambert JC, Heath S, Even G, Campion D, Sleegers K, Hiltunen M, Combarros O, Zelenika D, Bullido MJ, Tavernier B, Letenneur L, Bettens K, Berr C, Pasquier F, Fiévet N, Barberger-Gateau P, Engelborghs S, De Deyn P, Mateo I, Franck A, et al. (2009) Genome-wide association study identifies variants at CLU and CR1 associated with Alzheimer's disease. Nat Genet 41:1094-1099.

Lancaster TM, Baird A, Wolf C, Jackson MC, Johnston SJ, Donev R, Thome J, Linden DE (2011) Neural hyperactivation in carriers of the Alzheimer's risk variant on the clusterin gene. Eur Neuropsychopharmacol. Advance online publication. Retrieved March 2011. doi:10.1016/j.euroneuro.2011.02.001.

Meyer-Lindenberg A (2009) Neural connectivity as an intermediate phenotype: brain networks under genetic control. Hum Brain Mapp 30:1938-1946.

Meyer-Lindenberg A, Weinberger DR (2006) Intermediate phenotypes and genetic mechanisms of psychiatric disorders. Nat Rev Neurosci $7: 818-827$

Rempel-Clower NL, Barbas H (2000) The laminar pattern of connections between prefrontal and anterior temporal cortices in the Rhesus monkey is related to cortical structure and function. Cereb Cortex 10:851-865.

Schacter DL, Alpert NM, Savage CR, Rauch SL, Albert MS (1996) Conscious recollection and the human hippocampal formation: evidence from positron emission tomography. Proc Natl Acad Sci U S A 93:321-325.

Seshadri S, Fitzpatrick AL, Ikram MA, DeStefano AL, Gudnason V, Boada M, Bis JC, Smith AV, Carassquillo MM, Lambert JC, Harold D, Schrijvers EM, Ramirez-Lorca R, Debette S, Longstreth WT Jr, Janssens AC, Pankratz VS, Dartigues JF, Hollingworth P, Aspelund T, et al. (2010) Genome-wide analysis of genetic loci associated with Alzheimer disease. Jama 303:1832-1840.

Sheline YI, Morris JC, Snyder AZ, Price JL, Yan Z, D’Angelo G, Liu C, Dixit S, Benzinger T, Fagan A, Goate A, Mintun MA (2010) APOE4 allele disrupts resting state fMRI connectivity in the absence of amyloid plaques or decreased CSF Abeta42. J Neurosci 30:17035-17040.

Simons JS, Spiers HJ (2003) Prefrontal and medial temporal lobe interactions in long-term memory. Nat Rev Neurosci 4:637-648.

Sleegers K, Lambert JC, Bertram L, Cruts M, Amouyel P, Van Broeckhoven C (2010) The pursuit of susceptibility genes for Alzheimer's disease: progress and prospects. Trends Genet 26:84-93. 